\title{
KIT pathway upregulation predicts dasatinib efficacy in acute myeloid leukemia
}

\section{Malani, Disha}

2020-07-17

Malani , D , Yadav , B , Kumar , A , Potdar , S , Kontro , M , Kankainen , M , Javarappa , K K , Porkka , K, Wolf , M , Aittokallio , T, Wennerberg , K, Heckman , C A, Murumägi , A \& Kallioniemi , O 2020 , ' KIT pathway upregulation predicts dasatinib efficacy in acute myeloid pÿleukemia ' , Leukemia , vol. 34 , no. 10 , 34 , pp. 27802784 . https://doi.org/10.1038/s41375-020-0978-7

http://hdl.handle.net/10138/323955

https://doi.org/10.1038/s41375-020-0978-7

acceptedVersion

Downloaded from Helda, University of Helsinki institutional repository.

This is an electronic reprint of the original article.

This reprint may differ from the original in pagination and typographic detail.

Please cite the original version. 
1 KIT pathway upregulation predicts dasatinib efficacy in acute myeloid leukemia

3 Disha Malani $^{1}$, Bhagwan Yadav $^{1}$, Ashwini Kumar ${ }^{1}$, Swapnil Potdar $^{1}$, Mika Kontro ${ }^{1,2,3}$,

4 Matti Kankainen ${ }^{2}$, Komal K. Javarappa ${ }^{1}$, Kimmo Porkka ${ }^{2,3}$, Maija Wolf ${ }^{1}$, Tero

5 Aittokallio $^{1,4}$, Krister Wennerberg ${ }^{1,5}$, Caroline A. Heckman ${ }^{1}$, Astrid Murumägi ${ }^{1}$, Olli

6 Kallioniemi ${ }^{1,6}$

7

8 1. Institute for Molecular Medicine Finland (FIMM), HiLIFE, University of Helsinki, $9 \quad$ Helsinki, Finland

10 2. Hematology Research Unit Helsinki, University of Helsinki, Helsinki, Finland

11 3. Department of Hematology, University Hospital Comprehensive Cancer Center, $12 \quad$ Helsinki, Finland

13 4. Department of Cancer Genetics, Institute for Cancer Research, Oslo University 14 Hospital, and Oslo Centre for Biostatistics and Epidemiology, University of Oslo, 15 Oslo, Norway

16 5. Biotech Research \& Innovation Centre, BRIC and Novo Nordisk Foundation Center 17 for Stem Cell Biology, DanStem, University of Copenhagen, Copenhagen, Denmark 18 6. Science for Life Laboratory, Department of Oncology and Pathology, Karolinska 19 Institutet, Solna, Sweden

21 Keywords: acute myeloid leukemia, dasatinib, molecular profiling, high-throughput 22 drug testing, pathway dependency, RNA-sequencing 
Corresponding author: Disha Malani

24 Institute for Molecular Medicine Finland (FIMM), University of Helsinki,

25 Address: Biomeducum 2U, Tukholmankatu 8,

26 FIN-00290 Helsinki, Finland

27 Email: disha.malani@helsinki.fi

28 Phone: +358440341101

30 Text word count: $1486 / 1500$

31 Figures: $2 / 2$

32 References: $15 / 15$

33 Supplementary Figures: 7

34 Supplementary Tables: 9

\section{Conflicts of Interest:}

37 The authors declare no competing financial interests for this work. The senior authors

38 have received collaborative research grants for other projects as listed: OK received 39 research funding from Vinnova for collaboration between Astra-Zeneca, Takeda, 40 Pelago and Labcyte. OK is also a board member and a co-founder of Medisapiens and 41 Sartar Therapeutics and has received a royalty on patents licensed by Vysis-Abbot. KP 42 received honoraria and research funding from Bristol-Myers Squibb, Celgene, Novartis 43 and Pfizer. $\mathrm{CH}$ received honoraria from Celgene, Novartis and Roche and research 44 funding from Celgene, Novartis, Oncopeptides, Pfizer and the IMI2 project 45 HARMONY. KW received research funding from Novartis and Pfizer. MKo: research 46 funding from AbbVie. 
47 Acute myeloid leukemia (AML) is an aggressive malignant disease with a poor 48 prognosis. Although the recent approval of several new targeted drugs provides new 49 treatment options for subsets of patients, molecular heterogeneity in AML still poses a 50 major challenge for the patient treatment ${ }^{1}$. Novel treatments are needed to cover the 51 entire molecular spectrum of the disease. We and others have previously shown that 52 functional ex vivo drug testing of patient-derived primary AML cells provides 53 additional insights on the potential utility of e.g. dasatinib, venetoclax and 54 dexamethasone for treatment of subsets of AML patients ${ }^{2-5}$. However, in most cases, 55 the mechanism of action and the specific subgroups and biomarkers associated with the 56 drug effects have remained unknown. Cell lines originating from different cancer types 57 have provided valuable information about the complexity of cancer at the genomic, 58 epigenomic, transcriptomic and drug response level ${ }^{6-9}$, including observations in $59 \mathrm{AML}^{10,11}$. However, the representability of the AML cell lines of patient AML 60 specimens has remained unclear. Here, we aimed to i) integrate and compare 61 pharmacological profiles between AML cell lines and patient samples to identify 62 differential drug sensitivities; and ii) define molecular determinants and biomarkers of 63 drug response by the integration of in vitro, ex vivo and in vivo patient data, focusing 64 on KIT pathway and its inhibitors. We compared drug response profiles between ex vivo AML patient samples $(n=45)$

67 (Table S1) and established AML cell lines $(\mathrm{n}=28)$ (Table S2) using high-throughput drug sensitivity and resistance testing (DSRT) with 290 approved and investigational 69 oncology compounds (Table S3). Drug responses were quantified as drug sensitivity 70 score (DSS) ${ }^{12}$. Briefly, DSS is a quantitative measurement of drug response to define 71 drug efficacy using dose-response parameters. The differential drug sensitivity score 
(dDSS) for each sample was based on comparing the DSS for that sample with the mean over all patient samples (Table S4) or mean over all cell lines (Table S5). The mutation spectrum of the AML cell lines was obtained from cancer gene panel sequencing (Table S6) while exome sequencing was applied to the patient samples and analyzed as described previously ${ }^{2}$. RNA-seq data for the AML patient specimens were generated and analyzed as described previously ${ }^{13}$ and for the AML cell lines obtained from a published study ${ }^{9}$.

We first compared drug sensitivity patterns between ex vivo AML patient samples and cell lines using the Wilcoxon rank-sum test to reveal differential sensitivity across all drugs (Table S7). Higher efficacy for many cytotoxic chemotherapeutic drugs in the cell lines (Fig 1a) was likely due to the higher proliferation rate during drug testing as compared to the patient cells (Fig S1a). Therefore, we focused on targeted drugs exhibiting higher efficacy in the patient samples. We observed significantly higher efficacy of both multi tyrosine kinase inhibitors masitinib and dasatinib (Fig S1b) in patient samples compared to the cell lines. These two drugs inhibit KIT among other target genes, and hence belong to the same drug class, thus increasing the confidence of the finding.

We next explored drug sensitivities of targeted drugs in relation to common driver mutations in AML. Some of the common AML-related mutations e.g., KRAS, NRAS, EZH2, TP53 were more common in cell lines whereas FLT3-ITD, DNMT3A, NPM1, IDH1, IDH2 mutations were more common in the patient samples (Fig S2).

95 Unsupervised hierarchical clustering of 114 targeted sensitive drugs demonstrated mutation-based subgroups among the patient samples (Fig S3a) and the cell lines (Fig 
114 Next, we sought to identify molecular biomarkers for dasatinib sensitivity. We found

115 no significant association between the ex vivo efficacy of dasatinib and the presence of

$\mathrm{S} 3 \mathrm{~b})$. Dasatinib was found to be in the same cluster as other tyrosine kinase inhibitors e.g. axitinib, imatinib, masitinib in patient samples. However, the dasatinib response was distinct from the other tyrosine kinase inhibitors in AML cell lines.

Next, the percentage of responders was calculated for individual drugs in both cell lines and patient samples to estimate drug efficacy in individual samples. A drug was defined as effective if its dDSS value exceeded the $95 \%$ quantile (8.5) of the overall dDSS distribution (Fig S4a). We then compared the percentage of responders (dDSS $\geq 8.5$ ) in both patient samples and cell lines across 224 targeted drugs (Fig 1b). The analysis revealed that dasatinib was one of the drugs that exhibited remarkable differential sensitivity in AML patient samples compared to the cell lines. We found $20 \%$ (9 out of 45) AML patient samples and 11\% (3 out of 28) of cell lines were sensitive to dasatinib. Next, we assessed whether the dasatinib sensitivity was dependent on cell viability during assay but found no remarkable association using the patient samples (Figure S4b). Thus, dasatinib exhibited consistently higher efficacy in the patient samples as compared to the cell lines.

$$
\text { any of the common driver mutations in the AML patient samples (Figure S5a). None }
$$
of the AML patient samples had a mutation in the dasatinib target gene KIT (Fig S3a). Neither did we see any associations of ex vivo dasatinib response with clinical features of the disease (Figure S5b). We analyzed gene expression levels of dasatinib target proteins and found no significant correlation with dasatinib response either in the patient samples (Fig S5c) or in cell lines (Fig S5d). We then analyzed gene expression 
122 levels in terms of deregulated pathways using gene set variation analysis (GSVA) ${ }^{14}$ and 123 applied FDR to define KIT enrichment scores and their confidence levels (Fig 2a, Table 124 S8). The KIT pathway gene signature derived from the REACTOME pathway database 125 included 16 genes; FYN, KITLG, CBL, SH2B3, PTPN6, SOS1, PRKCA, KIT, SH2B2, 126 SOCS6, YES1, GRB2, LCK, SOCS1, SRC, LYN. The majority of the genes encode for 127 tyrosine kinases and signaling adaptor proteins (Table S9). Comparison of dasatinib 128 sensitive and non-sensitive AML patients showed that the KIT pathway upregulation 129 was a strong predictor for ex vivo dasatinib efficacy in AML (Fig 2b), stronger than the 130 expression of any of the dasatinib targets alone. While KIT pathway upregulation is a 131 stronger molecular determinant of ex vivo dasatinib efficacy than mutations or clinical 132 features, its potential utility to assign dasatinib treatment for AML needs additional 133 information.

135 Given the strong relationship between dasatinib sensitivity and KIT pathway 136 upregulation, we then assessed if this effect is mediated through KIT as one of the 137 targets. KIT gene is one of the sixteen genes of the KIT pathway. KIT (CD117) is a 138 receptor tyrosine kinase expressed on the cell surface. We investigated the effect of 139 dasatinib treatment on KIT protein expression and the induction of apoptosis to further 140 define the effects of dasatinib in AML cell lines. The KIT targeting drugs dasatinib, 141 masitinib, axitinib and imatinib (Fig S6a) was strongly effective in GDM-1, where the 142 KIT pathway was also strongly and significantly upregulated (Fig S6b). We found 143 reduced surface levels of KIT in dasatinib-treated GDM-1 cells as well as in KIT144 mutant KASUMI-1 cells (positive control). In contrast, no such effect was seen after 145 dasatinib treatment in MOLM-16 cells that are dasatinib-resistant and have no KIT 146 pathway upregulation (Fig 2c). We also observed increased intracellular levels of 
147 cleaved caspase 3 in KASUMI-1 and GDM-1 upon dasatinib treatment, compared to 148 the responses in MOLM-16 cells (Fig 2d), indicating that dasatinib treatment-induced 149 apoptosis (Fig S6c). Our findings are consistent with an earlier report suggesting 150 dasatinib treatment reduces cell surface expression of KIT due to endocytosis in AML 151 cells $^{15}$. These results, therefore, suggest that the effects of dasatinib on AML cell 152 viability and apoptosis could be mediated via the downregulation of the KIT protein. 153 However, the overall gene expression profiles linked to the entire KIT pathway 154 provided the strongest value as a drug response biomarker for predicting dasatinib 155 response.

157 We also assessed KIT pathway enrichment scores in three chemo-refractory AML 158 patients (AML_11, AML_36 and AML_41) treated with dasatinib to further explore 159 the clinical relevance of the finding. Dasatinib was selected for clinical translation as a 160 drug of choice for these patients based on leukemia-selective dasatinib response in $e x$ 161 vivo DSRT (Fig S7a) at Helsinki University Hospital ${ }^{2}$. In two patients characterized by 162 ex vivo dasatinib sensitivity and significant KIT pathway upregulation, dasatinib 163 treatment led to complete remission (AML_36) and complete remission with 164 incomplete hematological recovery (AML_41). In patient case AML_11, which also 165 showed ex vivo dasatinib sensitivity but no upregulation of KIT pathway, no response 166 to dasatinib was observed during a short treatment period which was limited by toxic 167 side effects. (Fig 2e, S7b, c). Therefore, the patient data is also suggestive that KIT 168 pathway activity could define AML patients who are most likely to respond to and 169 benefit from dasatinib treatment. 
171 Taken together, the combination of in vitro, ex vivo and clinical data suggest that gene 172 expression-based KIT pathway upregulation could act as a biomarker of dasatinib 173 efficacy in AML. We suggest that the upregulation of the KIT pathway in combination 174 with ex vivo dasatinib sensitivity testing could help to define patients who are most 175 likely to benefit from this treatment, a hypothesis to be tested in the form of a clinical 176 study. 


\section{Acknowledgements}

We are grateful to the patients who donated samples to the study and thank the FIMM High Throughput Biomedicine Unit and Breeze DSRT data analysis pipeline teams for their support. The research was funded by the Finnish Cultural Foundation (DM), the Blood Disease Foundation Finland (DM), Finnish Hematology Association SHY (DM, AK), Ida Montinin Foundation (DM), EMBO short-term fellowship (AK), the Academy of Finland (Center of Excellence for Translational Cancer Biology; grants 310507, 313267, 326238 to TA; 277293 to KW; iCAN Digital Precision Cancer Medicine Flagship grant 1320185 to TA, CH), Cancer Society of Finland (DM, AK, OK, TA, KW, CH), Sigrid Jusélius Foundation (to OK, KP, TA and KW), EU Systems Microscopy (FP7) and TEKES/Business Finland (to OK and KP), Novo Nordisk Foundation (to KW; NNF17CC0027852). OK supported by Knut and Alice Wallenberg Foundation, Swedish Foundation for Strategic Research, VR environment grant. MK supported by University of Helsinki and Finnish Medical Foundation.

\section{Authorship contribution}

DM, AM and OK designed the study. DM and AM performed drug testing experiments.

DM, BY and AK analyzed and visualized the data. DM generated hypotheses and interpreted results. DM and KJ designed and performed flow cytometry experiments. MK performed cell line variant calling and SP performed drug response data quality analysis. DM wrote the manuscript. MKo and KP obtained ethical permits, collected clinical samples and administered therapies. KP, MKo, TA, MW, KW, CH, AM and OK provided critical review. All authors contributed to and approved the final version of the manuscript. 
Figure 1. Dasatinib has high sensitivity in AML patient samples compared to AML

cell lines. A) Comparison of 290 drug responses between 45 AML patient samples and 28 AML cell lines. The median values of drugs plotted on the $\mathrm{x}$-axis and negative $\log 10$ of p-values plotted on the y-axis, where the statistical significance was calculated using 206 the Wilcoxon rank-sum test. Dot colors indicate significant drugs (FDR <0.1) with high sensitivity in patient samples (orange) and cell lines (blue). B) Correlation of percent responders for 224 targeted drugs between 28 AML cell lines (x-axis) and 45 AML patient samples (y-axis). The highlighted drugs depict outliers based on percent responders above 15 percentage for AML patient samples and below 15 percentage for 211 AML cell lines (the red dotted lines).

214 pathway enrichment scores aligned to dasatinib response (dDSS). The dotted line 215 represents sensitivity cut-off at 8.5 based on overall dDSS distribution. The asterisk marks represent significance levels as false discovery rates (FDR). B) KIT pathway 217 enrichment scores in dasatinib sensitive $(\mathrm{dDSS}>8.5)$ and non-sensitive $(\mathrm{dDSS}<8.5)$ 218 patient samples. C) Flow cytometry analysis represents the percentage of KIT positive 219 cells in untreated (DMSO control) and 500nM dasatinib treated KASUMI-1, GDM-1 220 and MOLM-16 cells. D) Flow cytometry analysis represents the percentage of cleaved 221 caspase 3 positive cells in untreated (DMSO control) and 500nM dasatinib treated 222 KASUMI-1, GDM-1 and MOLM-16 cells. E) Ex vivo dasatinib response and matched 223 KIT pathway in three AML patient cases who were given dasatinib treatment. The 224 clinical outcomes of the treatment defined as a resistant disease (RD), complete 
225 remission (CR) and complete remission with incomplete hematological recovery (CRi) 226 for all patients. 


\section{References}

1. Döhner H, Estey E, Grimwade D, Amadori S, Appelbaum FR, Büchner T, et al.

2. Pemovska T, Kontro M, Yadav B, Edgren H, Eldfors S, Szwajda A, et al. Individualized Systems Medicine Strategy to Tailor Treatments for Patients with Chemorefractory Acute Myeloid Leukemia. Cancer Discovery 2013; 3(12): 1416-1429.

3. Tyner JW, Tognon CE, Bottomly D, Wilmot B, Kurtz SE, Savage SL, et al. Functional genomic landscape of acute myeloid leukaemia. Nature 2018 2018/10/17.

4. Snijder B, Vladimer GI, Krall N, Miura K, Schmolke A-S, Kornauth C, et al. Image-based ex-vivo drug screening for patients with aggressive haematological malignancies: interim results from a single-arm, open-label, pilot study. The Lancet Haematology 2017; 4(12): e595-e606.

5. Malani D, Murumagi A, Yadav B, Kontro M, Eldfors S, Kumar A, et al. Enhanced sensitivity to glucocorticoids in cytarabine-resistant AML. Leukemia 2017 May; 31(5): 1187-1195.

6. Wilding JL, Bodmer WF. Cancer cell lines for drug discovery and development. Cancer Research 2014; 74(9): 2377-2384.

7. Garnett MJ, Edelman EJ, Heidorn SJ, Greenman CD, Dastur A, Lau KW, et al. Systematic identification of genomic markers of drug sensitivity in cancer cells. Nature 2012; 483(7391): 570-575.

8. Barretina J, Caponigro G, Stransky N, Venkatesan K, Margolin AA, Kim S, et al. The Cancer Cell Line Encyclopedia enables predictive modelling of anticancer drug sensitivity. Nature 2012; 483(7391): 603-607.

9. Ghandi M, Huang FW, Jané-Valbuena J, Kryukov GV, Lo CC, McDonald ER, et al. Next-generation characterization of the Cancer Cell Line Encyclopedia. Nature 2019; 569(7757): 503-508.

10. Lee S-I, Celik S, Logsdon BA, Lundberg SM, Martins TJ, Oehler VG, et al. A machine learning approach to integrate big data for precision medicine in acute myeloid leukemia. Nature Communications 2018; 9(1): 42.

11. Iorio F, Knijnenburg TA, Vis DJ, Bignell GR, Menden MP, Schubert M, et al. A Landscape of Pharmacogenomic Interactions in Cancer. Cell 2016 Jul 28; 166(3): 740-754.

12. Yadav B, Pemovska T, Szwajda A, Kulesskiy E, Kontro M, Karjalainen R, et al. Quantitative scoring of differential drug sensitivity for individually optimized anticancer therapies. Scientific reports 2014; 4: 5193. 
13. Kumar A, Kankainen M, Parsons A, Kallioniemi O, Mattila P, Heckman CA. The impact of RNA sequence library construction protocols on transcriptomic profiling of leukemia. BMC genomics 2017; 18(1): 629-629.

14. Hänzelmann S, Castelo R, Guinney J. GSVA: gene set variation analysis for microarray and RNA-Seq data. BMC Bioinformatics 2013; 14(1): 7.

15. Heo S-K, Noh E-K, Kim JY, Jeong YK, Jo J-C, Choi Y, et al. Targeting c-KIT (CD117) by dasatinib and radotinib promotes acute myeloid leukemia cell death. Scientific Reports 2017; 7(1): 15278. 
Figure 1

A

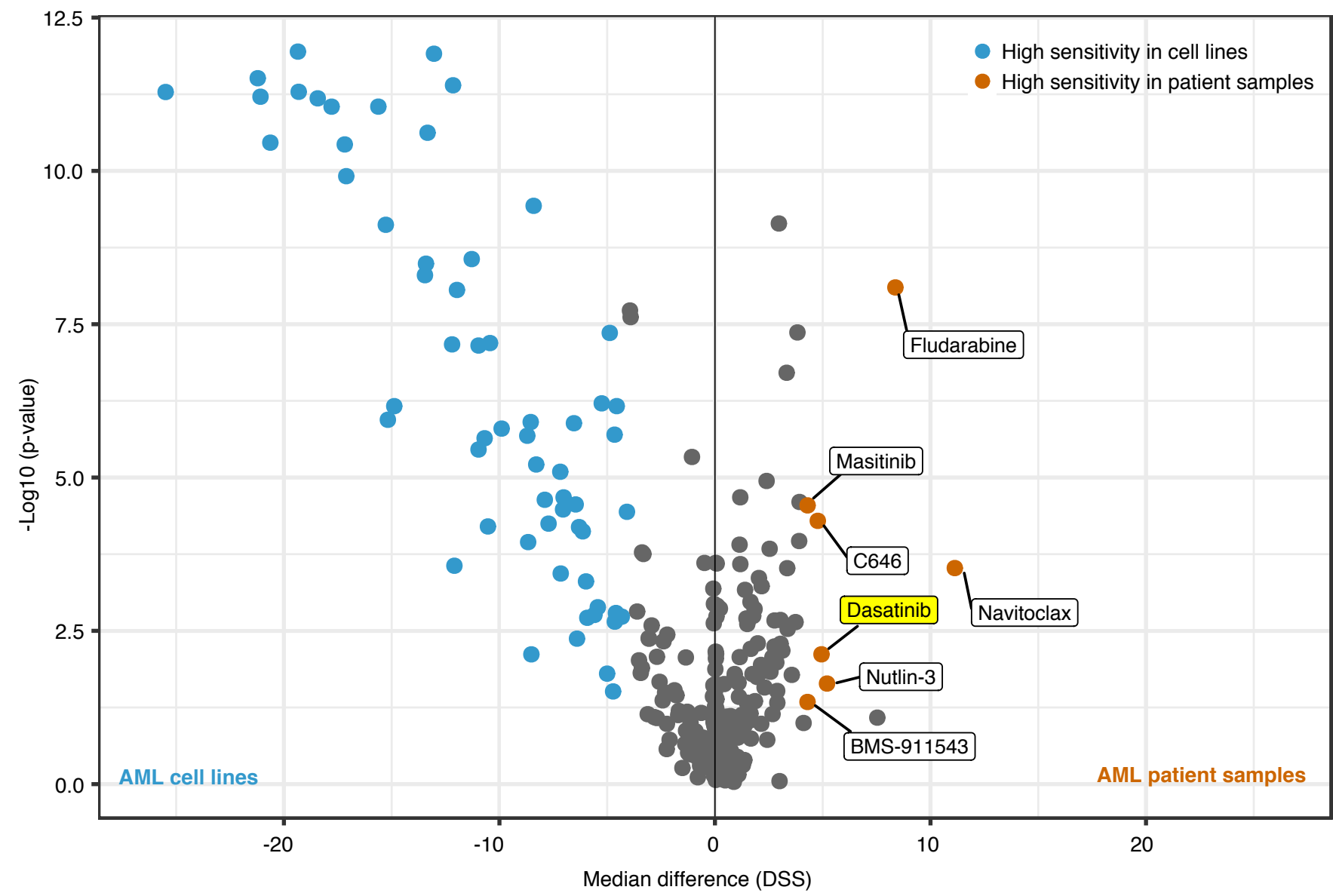

B

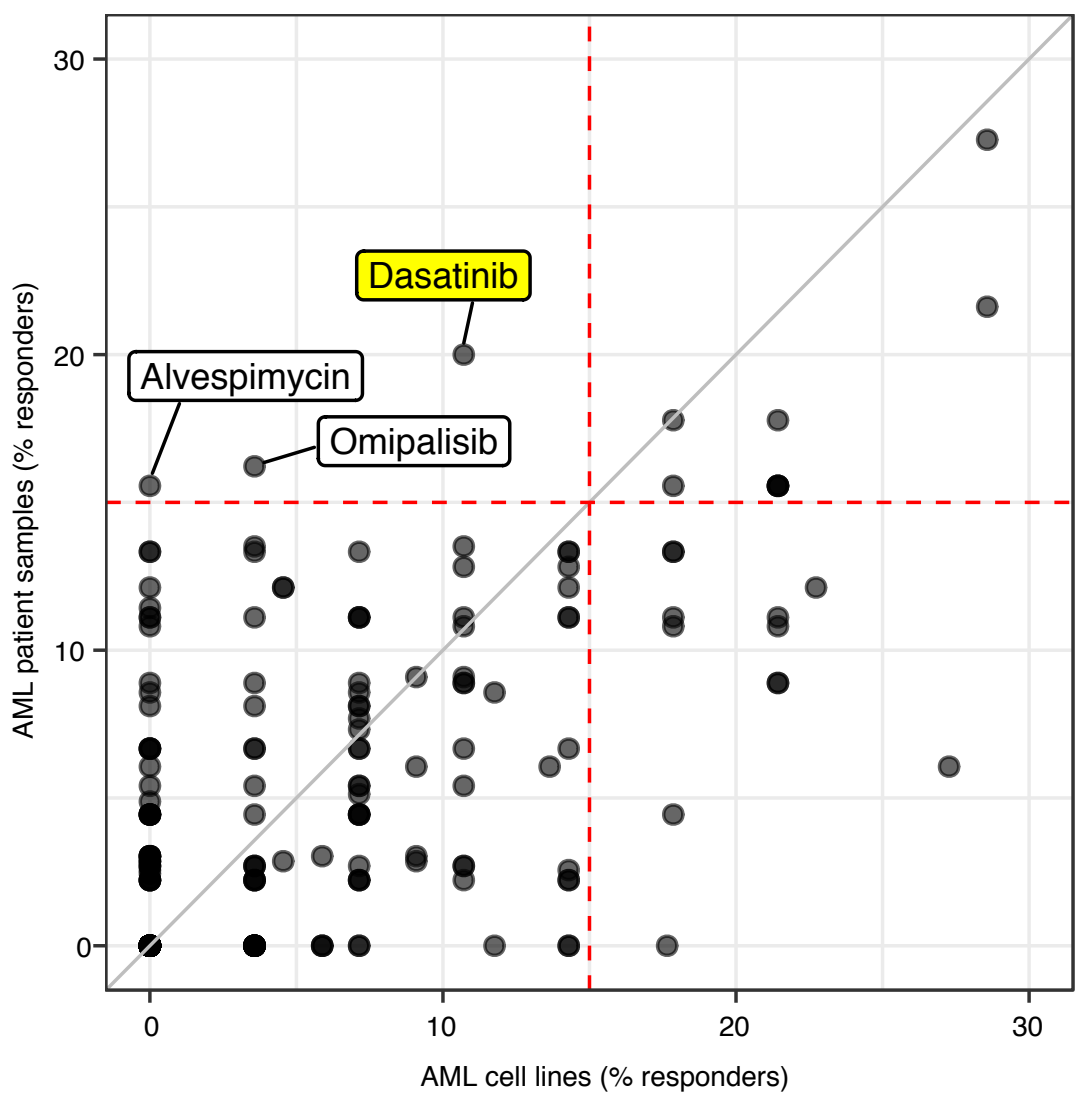


Figure 2

A

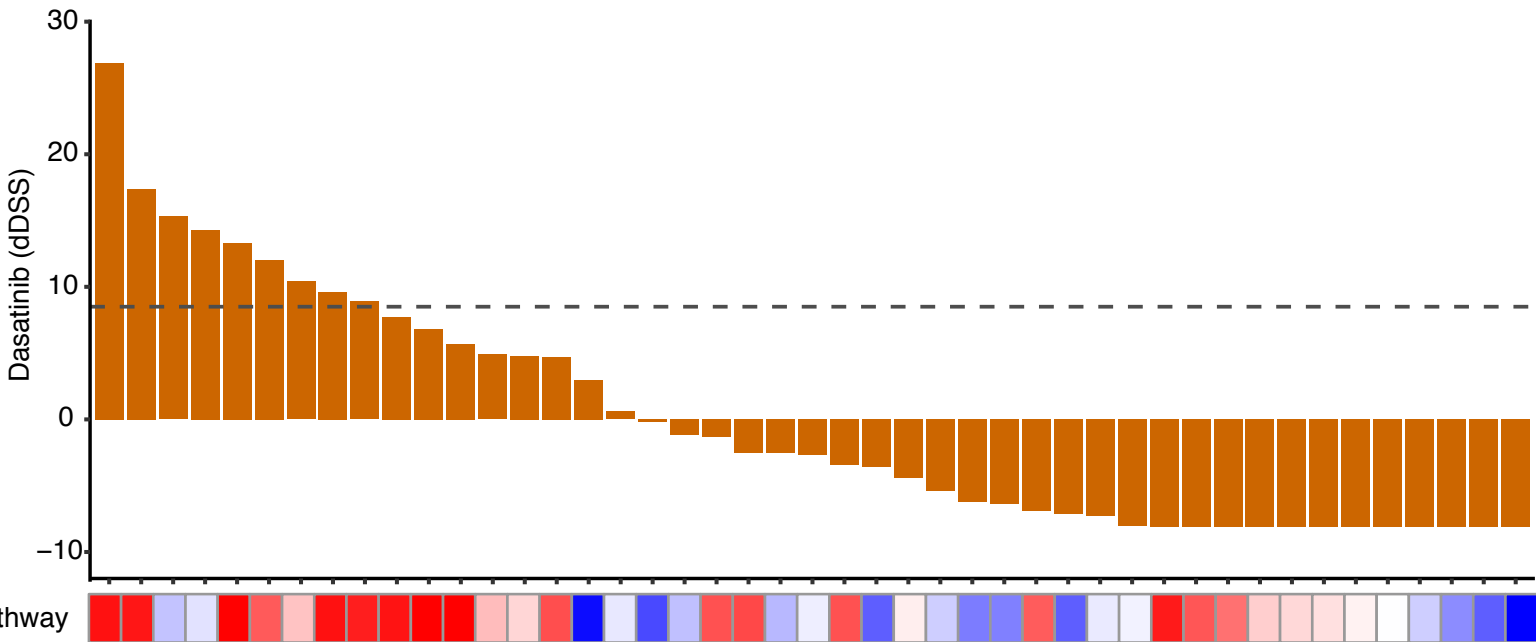

KIT pathway

KIT pathway $* * x$

(FDR)

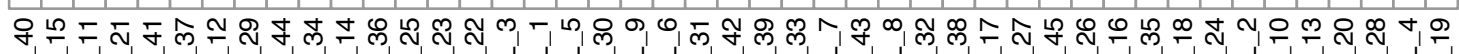

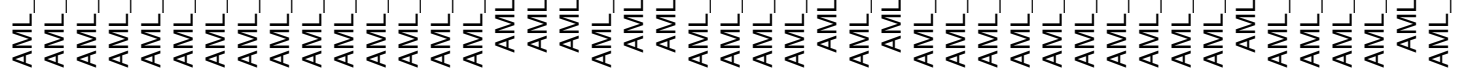

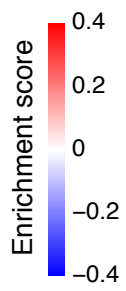

B

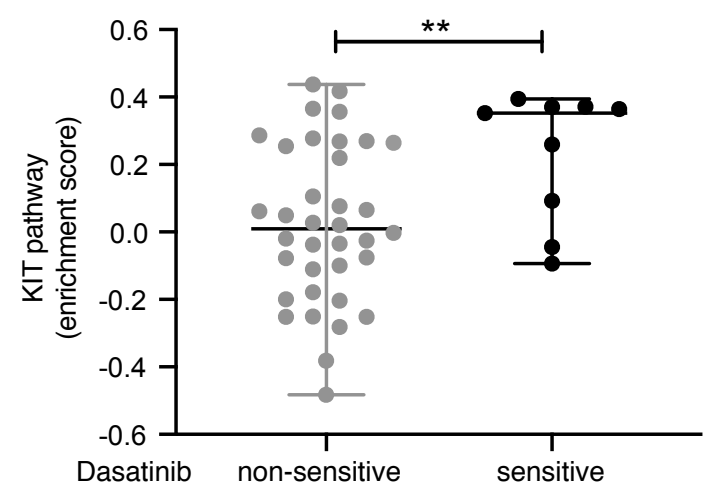

D

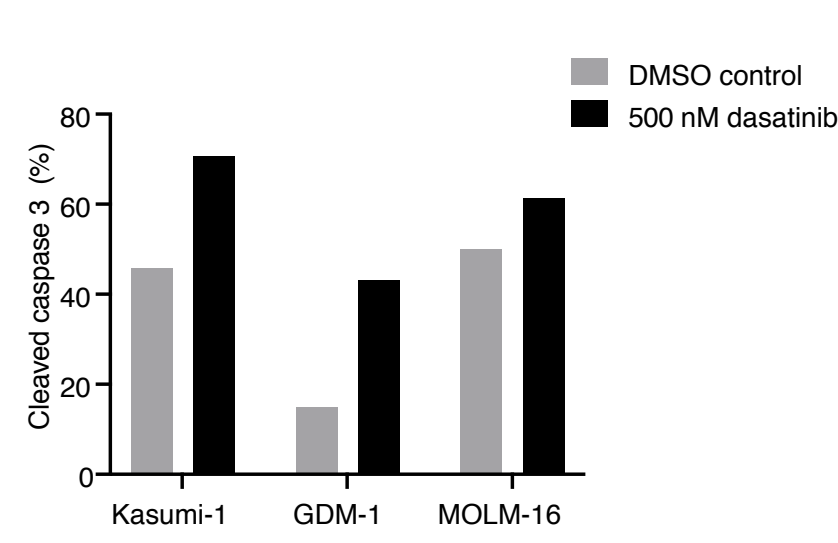

C

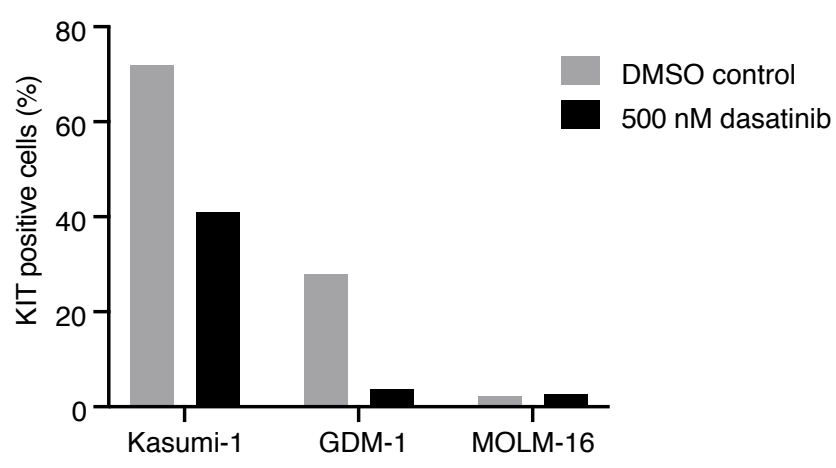

E

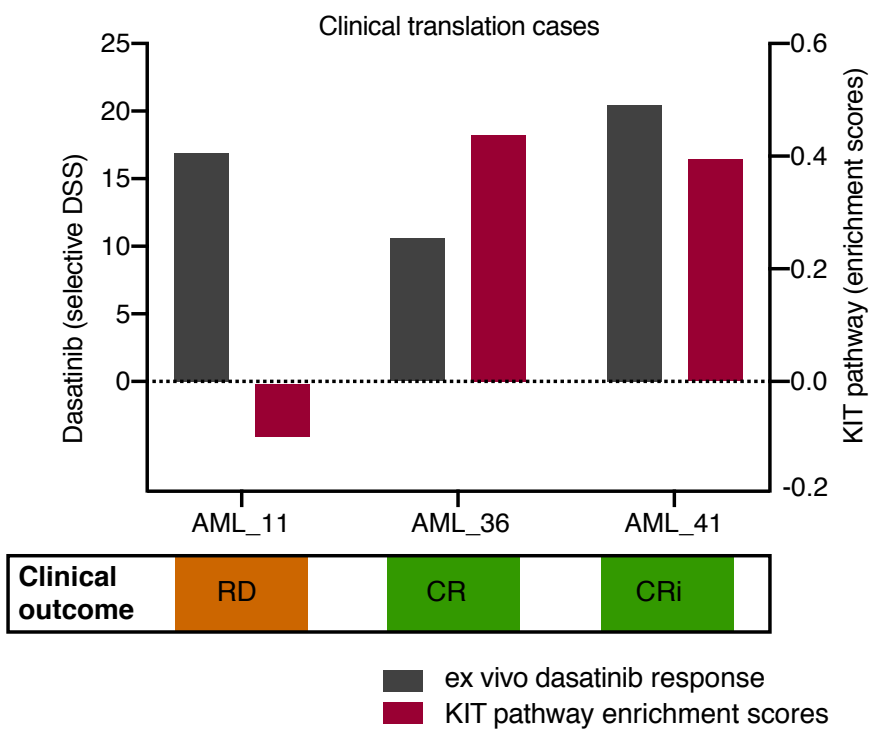


1 KIT pathway upregulation predicts dasatinib efficacy in acute myeloid leukemia

2

3 Disha Malani ${ }^{1}$, Bhagwan Yadav $^{1}$, Ashwini Kumar ${ }^{1}$, Swapnil Potdar ${ }^{1}$, Mika Kontro ${ }^{1,2,3}$, Matti

4 Kankainen $^{2}$, Komal K. Javarappa ${ }^{1}$, Kimmo Porkka ${ }^{2,3}$, Maija Wolf ${ }^{1}$, Tero Aittokallio ${ }^{1,4}$, Krister

5 Wennerberg ${ }^{1,5}$, Caroline A. Heckman ${ }^{1}$, Astrid Murumägi ${ }^{1}$, Olli Kallioniemi ${ }^{1,6}$

6

7 1. Institute for Molecular Medicine Finland (FIMM), HiLIFE, University of Helsinki, Helsinki, $8 \quad$ Finland

9 2. Hematology Research Unit Helsinki, University of Helsinki, Helsinki, Finland

10 3. Department of Hematology, University Hospital Comprehensive Cancer Center, Helsinki, Finland

11 4. Department of Cancer Genetics, Institute for Cancer Research, Oslo University Hospital, and Oslo Centre for Biostatistics and Epidemiology, University of Oslo, Oslo, Norway

13 5. Biotech Research \& Innovation Centre, BRIC and Novo Nordisk Foundation Center for Stem Cell 14 Biology, DanStem, University of Copenhagen, Copenhagen, Denmark

15 6. Science for Life Laboratory, Department of Oncology and Pathology, Karolinska Institutet, Solna, 16 Sweden 


\section{Supplementary figure legends:}

Figure S1. A) Percentage increment at $72 \mathrm{~h}$ compared to $0 \mathrm{~h}$ in terms of luminescence-based cell viability during drug testing experiment for ex vivo AML patient samples and AML cell lines. B) Comparison of drug sensitivity scores (DSS) for dasatinib between 45 AML patient samples and 28 AML cell lines. The Wilcoxon rank-sum test was applied to calculate p-value.

Figure S2. Mutation frequencies of 23 AML related driver genes in 45 patient samples and in 28 AML cell lines where FLT3-ITD represents internal tandem duplication and FLT3-PM represents point mutations in FLT3 gene.

Figure S3. Unsupervised hierarchical clustering responses of 114 targeted sensitive drugs and A) 45 AML patient samples or B) 28 AML cell lines. The key AML related mutations annotated for both patient samples and cell lines.

Figure S4. A) Distribution of differential drug sensitivity scores (dDSS) of 290 drug responses from 45 AML patient samples and 28 AML cell lines. Significant dDSS cut off 8.5 was defined as a $95 \%$ quantile of the overall distribution. B) Upper panel depicts response to dasatinib (dDSS and lower panel illustrates cell viability measured during drug testing assay in absence of drug ( 72 hours) for 37 AML patient samples.

Figure S5. A) Comparison of dasatinib response (DSS) between wild type and mutated samples for FLT3-PM (point mutation), FLT3-ITD (internal tandem duplication), NPM1, NRAS, PTPN11, DNMT3A using nonparametric Mann-Whitney test, where ns represents non-significant p-values. B) Pearson correlation of age with dasatinib response. Comparison of the clinical features with dasatinib responses using Mann-Whitney test, where ns represents non-significant p-values. C) Expression of 
42 dasatinib target genes aligned to dasatinib response in 45 AML patient samples. The bar plot on the

43 right side depicts the Pearson correlation values between dasatinib response (dDSS) and RNA-seq

44 derived expression values ( $\log 2$ count per million $(\mathrm{CPM}))$ of individual genes D) Expression $\left(\log _{2}\right.$

45 CPM) of dasatinib target genes aligned to dasatinib response (dDSS) in 21 AML cell lines.

46

47 Figure S6. A) dDSS for tyrosine kinase inhibitors (dasatinib, masitinib, axitinib and imatinib) in 48 AML cell lines GDM-1, KASUMI-1 and MOLM-16. B) Enrichment score for the KIT pathway 49 aligned to dasatinib response in 21 AML cell lines. The asterisk marks represent significance as false 50 discovery rates (FDR). c. The fluorescence signal of KIT antibody, cleaved caspase 3 antibody and 51 respective isotype control antibodies used for flow cytometry experiments.

52

53 Figure S7. A) Drug response profile of AML patient cases AML_11, AML_36 and AML_41

54 depicting the range of selective DSS (healthy bone marrow normalized DSS). Dasatinib is

55 highlighted in red. B) KIT pathway enrichment scores of AML patient samples where the patients 56 treated with dasatinib highlighted in red. c. Clinical information on drug treatment, disease status, 57 ex vivo dasatinib response and KIT pathway enrichment score for patients AML_11, AML_36 and 58 AML_41. 


\section{AML patient samples and cell lines}

The samples were collected from AML patients $(n=45)$ after signed informed consent using protocols approved by local ethical committees (approvals 239/13/03/00/2010 and 303/13/03/01/2011). Mononuclear cells were isolated from bone marrow aspirates and peripheral blood samples by FicollPaque (GE Healthcare) density gradient centrifugation. Twenty-eight AML cell lines were selected across French American British (FAB) classes, ranging from M0 to M7 subtypes. The cell lines were purchased from German collection of microorganisms and cell cultures (DSMZ), expect for HL-60 cell line that was purchased from American type tissue culture collection (ATCC) and HL-60_TB, a subline of HL60, was purchased from NCI-Frederick cancer DCTD tumor/cell line repository.

\section{Chemical compound collection}

The collection of 290 chemical compounds including 144 FDA (U.S. Food and Drug Administration) or EMA (European Medical Agency) approved drugs, 112 investigational compounds and 34 chemical probes. The collection consists of conventional chemotherapy drugs, kinase inhibitors, apoptosis modifiers, epigenetic modifiers, differentiating agents, metabolic modifiers, hormonal therapeutics, and immunomodulators. The annotation for mechanism of actions or molecular targets are given for each drug. The drugs were defined as sensitive if the dDSS was $>8.5$ for at least one cell line or patient sample.

\section{Drug Sensitivity and Resistance Testing (DSRT)}

All cell lines were cultured in vendor specified media, except for HL-60 and HL-60_TB, which were cultured in 90\% RPMI 1640 with 10\% FBS and Penicillin/Streptomycin. DSRT was performed with the cell lines and freshly isolated mononuclear cells from bone marrow or blood of 45 diagnostic and 
relapsed AML patients in mononuclear cell medium (Promocell) using the protocol described earlier ${ }^{1}$. Briefly, the 290 compounds were dissolved in DMSO and dispensed in 384 well cell culture plates using an Echo 550 acoustic liquid handling system (Labcyte). Each compound was plated at five increasing concentrations, covering 10 000-fold range, mostly from 1-10 000nM. The drug plates were stored in nitrogen gas pressurized pods (Roylan Development Ltd.) before use. Cell seeding density was optimized prior to DSRT experiments for each cell line whereas patient samples were plated at 10000 cells per well. Cells were plated in pre-drugged plates and incubated for $72 \mathrm{~h}$ at $37^{\circ} \mathrm{C}$ in $5 \% \mathrm{CO}_{2}$. Cell viability was measured using CellTiter Glo (Promega) reagent with PHERAstar FS (BMG Labtech) plate reader. Negative control DMSO and positive control benzethonium chloride were included in each assay plate for normalization of cell viability readouts (inhibition \%).

\section{Drug response data analysis}

The drug response data was analyzed using FIMM in-house Breeze pipeline ${ }^{2}$. Individual dose response curves and IC50 values were calculated for each drug using the FIMM DSRT data analysis pipeline. Drug sensitivity scores (DSS), a modified area under dose-response curve, was calculated as described previously ${ }^{3}$. Differential drug sensitivity scores (dDSS) were calculated for each drug separately by subtracting the mean DSS over all the samples or over all the cell lines from the samplespecific DSS. The dDSS were calculated separately for cell lines and patient samples. Drug response data quality was assessed with Z-prime score, where variation was calculated between multiple positive and negative controls from the same plate.

Wilcoxon rank-sum test was performed with DSS to identify the drugs with significant differential efficacy between AML patient samples compared to the cell lines. The false discovery rates (FDR) were calculated using Benjamini \& Hochberg method ${ }^{4}$. Median DSS difference <-4 or $>4$ and FDR $<0.1$ were considered significant for differential efficacy of drugs. The sensitivity was defined if 
dDSS was above 8.5 , which corresponds to the $95 \%$ quantile of the overall dDSS distribution over

110 all the cell lines and patient samples. The percentage responders were calculated for a given drug

111 with percent sensitive cell lines or patient samples. Targeted sensitive drugs $(n=114)$ were defined if 112 dDSS value were above 8.5 in at least one of the samples, which corresponds to the $95 \%$ quantile of 113 the overall dDSS distribution over all the cell lines and patient samples. Unsupervised hierarchical 114 clustering with complete linkage was performed using Euclidean distance for cell lines and patient 115 samples, and correlation distance for dDSS profiles of compounds.

\section{DNA sequencing and somatic variant analysis}

118 Exome sequencing was performed using DNA isolated from mononuclear cells of AML patient samples $(\mathrm{n}=45)$ and matching skin biopsies from the same patients using Agilent or Roche NimbleGen exome capture kits and a HiSeq 2500 instrument (Illumina). The data processing and variant calling was performed using same pipeline as described previously ${ }^{1}$. Genomic DNA was isolated from the cell lines ( $\mathrm{n}=28)$ using the DNeasy Blood and Tissue Kit (Qiagen). Massive paralleltargeted sequencing of 578 genes was performed using Nimblegen’s SeqCap EZ Designs kit (Roche). $2 \mu \mathrm{g}$ of DNA was used for library preparation, enrichment and sequencing using HiSeq 2500 (Illumina).

127 Genetic variants in each cell line were called and annotated as described earlier ${ }^{5}$. Briefly, variants were called using a modified GATK best-practice and annotated using Annovar tool against RefGene database. Subsequently, the variants called from the cell lines were filtered by removing variants not passing variant calling filters, not located in exonic or splice region synonymous SNVs and frameshift insertion and deletions. Furthermore, variants were removed if not found in hematopoietic malignancies in the COSMIC version 87 (https://cosmic-blog.sanger.ac.uk/cosmic-release-v87), or 
samples ${ }^{6}$. Finally, variants were removed if the variant's frequency was $>1 \%$ in gnomAD database of healthy individuals when considering all populations (https://gnomad.broadinstitute.org), if supported by $<10$ reads in total and $<2$ reads in either direction, having a variant allele frequency $<2 \%$, and having a strand odd ratio for $\mathrm{SNVs}_{2} \geq 3.00$, and strand odd ratio for indels $\geq 11.00$ and quality $<40$.

\section{RNA-sequencing}

RNA-sequencing was performed for 45 AML patient samples. Total RNA (2.5-5 $\mu \mathrm{g})$ was extracted from bone marrow or peripheral blood mononuclear cells from AML patients using the miRNeasy or AllPrep kit (Qiagen) and depleted of ribosomal-RNA (Ribo-ZeroTM rRNA Removal Kit, Epicentre) after purification, reverse transcribed to double stranded cDNA (SuperScriptTM Double- Stranded cDNA Synthesis Kit, Thermo Fisher). Library quality was evaluated on high sensitivity chips using the Agilent Bioanalyzer (Agilent Technologies). Paired-end sequencing with 100 bp read length was performed using HiSeq 2500 (Illumina) as described previously ${ }^{7}$.

\section{Gene expression analysis}

RNA-seq data pre-processing including, quality control, alignment, normalization, feature count and count per million (CPM) calculation were performed as described previously ${ }^{7}$. Briefly, Trimmomatic $^{8}$ was used to correct reads for low quality, Illumina adapters, and short read-length. Filtered pairedend reads were aligned to the human genome (GRCh38) using STAR aligner ${ }^{9}$ with the guidance of EnsEMBL v82 gene models. Feature counts were computed using SubRead ${ }^{10}$ R-package and converted to expression estimates using Trimmed Mean of M-values (TMM) normalization method

${ }^{11}$. Default parameters were used with exception that reads were allowed to be assigned to overlapping genome features in the feature counting. The published RNA-seq data (raw read counts) for 21 AML 
cell lines was obtained from the CCLE resource ${ }^{12}$. Raw reads were further normalized by TMM method and $\log _{2}$ CPM values were calculated similar to the patient samples.

\section{Pathway enrichment analysis}

To get the pathway enrichment scores, the gene expression values $\left(\log _{2} \mathrm{CPM}\right)$ for the protein coding genes were subjected to the gene set variation analysis (GSVA) using a R-package (GSVA version 1.18 .0$)^{13}$ for both 45 AML patient samples and 21 cell lines. The GSVA analysis calculates the relative enrichment of a gene set across the sample set. We applied GSVA analysis separately for patient samples and cell lines. The pathway gene set signatures were obtained from the Molecular Signatures Database (MSigDB) (https://www.gsea-msigdb.org/gsea/msigdb/collections.jsp\#C1) database version "c2.cp.reactome.v6.2.entrez.gmt". The canonical pathways were derived from REACTOME database ( $\mathrm{n}=674$ gene sets), where we specifically focused on "REACTOME_REGULATION_OF_KIT_SIGNALING” based on prior knowledge. A high enrichment score represents upregulated pathway, whereas low or negative scores represent downregulated pathways. We applied 1000 bootstrap iterations on GSVA scores in order to get the significance levels. Next, the p-values were adjusted across the patient sample or across the cell lines by applying Benjamini and Hochberg $(\mathrm{BH})$ method to get false discovery rates (FDR). The FDR < 0.05 was considered significant. The KIT pathway gene signature consists of 16 genes; FYN, KITLG, CBL, SH2B3, PTPN6, SOS1, PRKCA, KIT, SH2B2, SOCS6, YES1, GRB2, LCK, SOCS1, SRC, LYN. The KIT pathway enrichment scores ranged from -0.482 to 0.437 in case of AML patient samples $(n=45)$ and from -0.431 to 0.525 in AML cell lines $(n=21)$.

\section{Flow cytometry analysis}

Dasatinib and venetoclax were purchased from LC Laboratories and ChemieTek, respectively, and dissolved in DMSO to prepare $10 \mathrm{mM}$ stocks. The KASUMI-1, GDM-1 and MOLM-16 cells were 
treated with $100 \mathrm{nM}$ and $500 \mathrm{nM}$ dasatinib and $100 \mathrm{nM}$ venetoclax (a positive control for apoptosis induction) for $24 \mathrm{~h}$ in V-bottom 96 well plates (Nunc). After the incubation, the plate was centrifuged at $600 \mathrm{~g}$ for $4 \mathrm{~min}$ and supernatant was discarded. The cells were stained with 1:1000 dilution of zombie violet (BioLegend) as per the vendor's instructions. The cells were washed with $1 \mathrm{X}$ PBS and stained with 1:50 dilution of CD117(cKIT)-BV605 antibody (562687, BD Biosciences). The cells were washed with $1 \mathrm{X}$ PBS and fixed using $2.5 \%$ formaldehyde for $10 \mathrm{~min}$ at $37^{\circ} \mathrm{C}$ and permeabilized using $70 \%$ cold methanol for $20 \mathrm{~min}$ at $4^{\circ} \mathrm{C}$. Subsequently the washed cells were stained with 1:50 dilution of cleaved caspase 3-A647 antibody (9602, Cell Signaling Technology). The isotype control antibodies BV605 (562652, BD Biosciences) and A647(612599, BD Biosciences) were used at same concentration as the CD117 and cleaved caspase 3 antibodies. CD117 antibody stained UltraComp beads (01-2222-41, Invitrogen) and cleaved caspase 3 stained venetoclax treated Kasumi-1 cells were used for compensation. An iQue Plus (Intellicyte) flow cytometer was used to measure florescence of the stained cells and beads. The compensated data were analyzed using FlowJo ${ }^{\text {TM }}$ software (https://www.flowjo.com/).

\section{Clinical Translation in AML patients}

We have established leukemia precision medicine program to tailor targeted therapies based on top selective drug responses using functional testing and molecular profiles ${ }^{1}$. The program is a collaborative effort involving biologists, bioinformaticians and clinicians at Institute for Molecular Medicine Finland (FIMM) and Helsinki University Hospital. The program is primarily for end-stage chemo-refractory AML patients and in exceptional conditions for diagnostic primary AML patient cases. The treatment regimens comprised of approved non-AML drugs were used as a single agent or in combinations for clinical translation in individual AML patient cases, including serial therapy with different regimens in some of the patients. The drugs classified as signaling molecule inhibitors, immunomodulator, proteasome inhibitor and epigenetic modifier, were approved for cancer 
208 indications and patients were treated under off label compassionate usage. The regimens resulted in 209 either complete remission (CR), complete remission with incomplete hematological recovery (CRi) 210 or resistant disease (RD) defined by ELN2017 creiteria $^{14}$. Patient AML_11 was given dasatinib in 211 combination with azacytidine and was resistant to the therapy. Patient AML_36 was given dasatinib212 azacitidine therapy and the patient was MDR positive after the therapy, however the blast count 213 decreased after the therapy was defined as CRi as per ELN2017 criteria. Patient_41 was given 214 combination of dasatinib (multi-tyrosine kinase inhibitor), temsirolimus (mTOR inhibitor) and 215 sunitinib (tyrosine kinase inhibitor) and achieved complete remission with the therapy. We assumed 216 that dasatinib response associated with KIT pathway, considering ex vivo association and KIT being 217 one of the target genes, gave biological meaningful hypothesis.

219 Statistical Analyses

220 The statistical analyses were performed and figures were generated using Prism software version 8 221 (GraphPad) and R version 3.3 .3 (2017-03-06). Statistical dependence between two variables was 222 calculated using Pearson's correlation coefficient. The Wilcoxon rank-sum test was applied to assess 223 differences between drug responses. 


\section{References}

225 1. Pemovska T, Kontro M, Yadav B, Edgren H, Eldfors S, Szwajda A, et al. Individualized

2. Potdar S, Ianevski A, Mpindi JP, Bychkov D, Fiere C, Ianevski P, et al. Breeze: an integrated quality control and data analysis application for high-throughput drug screening. Bioinformatics 2020 Mar 2.

3. Yadav B, Pemovska T, Szwajda A, Kulesskiy E, Kontro M, Karjalainen R, et al. Quantitative scoring of differential drug sensitivity for individually optimized anticancer therapies. Scientific reports 2014; 4: 5193.

4. Benjamini Y, Hochberg Y. Controlling the false discovery rate: a practical and powerful approach to multiple testing. Journal of the Royal statistical society: series $B$ (Methodological) 1995; 57(1): 289-300.

5. Dufva O, Kankainen M, Kelkka T, Sekiguchi N, Awad SA, Eldfors S, et al. Aggressive natural killer-cell leukemia mutational landscape and drug profiling highlight JAK-STAT signaling as therapeutic target. Nature Communications 2018 2018/04/19; 9(1): 1567.

6. Tyner JW, Tognon CE, Bottomly D, Wilmot B, Kurtz SE, Savage SL, et al. Functional genomic landscape of acute myeloid leukaemia. Nature 2018 2018/10/17.

7. Kumar A, Kankainen M, Parsons A, Kallioniemi O, Mattila P, Heckman CA. The impact of RNA sequence library construction protocols on transcriptomic profiling of leukemia. $B M C$ genomics 2017; 18(1): 629-629.

8. Bolger AM, Lohse M, Usadel B. Trimmomatic: a flexible trimmer for Illumina sequence data. Bioinformatics 2014; 30(15): 2114-2120.

9. Dobin A, Davis CA, Zaleski C, Schlesinger F, Drenkow J, Chaisson M, et al. STAR: ultrafast universal RNA-seq aligner. Bioinformatics 2012; 29(1): 15-21.

10. Liao Y, Smyth GK, Shi W. The Subread aligner: fast, accurate and scalable read mapping by seed-and-vote. Nucleic Acids Research 2013; 41(10): e108-e108.

11. Robinson MD, Oshlack A. A scaling normalization method for differential expression analysis of RNA-seq data. Genome Biol 2010; 11(3): R25.

12. Ghandi M, Huang FW, Jané-Valbuena J, Kryukov GV, Lo CC, McDonald ER, et al. Nextgeneration characterization of the Cancer Cell Line Encyclopedia. Nature 2019; 569(7757): 503-508.

13. Hänzelmann S, Castelo R, Guinney J. GSVA: gene set variation analysis for microarray and RNA-Seq data. BMC Bioinformatics 2013; 14(1): 7. 
271 14. Döhner H, Estey E, Grimwade D, Amadori S, Appelbaum FR, Büchner T, et al. Diagnosis 272 and management of AML in adults: 2017 ELN recommendations from an international expert panel. Blood 2017; 129(4): 424-447. 